\title{
ÚLCERA MARGINAL PERFORADA POST BYPASS GÁSTRICO LAPAROSCÓPICO*
}

\author{
Drs. Andrés Marambio G. ${ }^{1}$, Mauricio Gabrielli N. ${ }^{1}$, Int. Juan Francisco de la Llera K. ${ }^{2}$, \\ Drs. Fernando Crovari E. ${ }^{1}$, Gustavo Pérez B. ${ }^{1}$, Luis Ibáñez A. ${ }^{1}$, Ricardo Funke H. ${ }^{1}$, \\ Fernando Pimentel M. ${ }^{1}$, Alex Escalona P. ${ }^{1}$, Enrique Norero M. ${ }^{1}$, Camilo Boza W. ${ }^{1}$ \\ 1 Departamento de Cirugía Digestiva. División de Cirugía. Pontificia Universidad Católica de Chile. \\ 2 Interno de Medicina. Facultad de Medicina. Pontificia Universidad Católica de Chile. \\ Santiago, Chile.
}

\begin{abstract}
\section{Perforated marginal ulcer after laparoscopic gastric bypass}

Introduction: Perforated marginal ulcer is a serious event that usually requires reoperation and is associated with morbidity and mortality. Characterization and management of these patients is still debated. Objective: To describe a series of patients subjected to a laparoscopic gastric bypass (LGBP) that evolved with a perforated marginal ulcer. Material and Methods: Records of patients undergoing a LGBP the last 10 years and evolved with a perforated marginal ulcer were retrospectively reviewed. Clinical features, treatment and perioperative morbidity and mortality were analyzed. Results: During this period 2,095 patients were subjected to a LGBP, 12 of them presented a perforated marginal ulcer, corresponding to 10 women and 2 men. Mean age was 39 (21-60) and mean body mass index at the time of initial surgery was 34 (29.3 to 38.6). Ten patients were smoker at the moment of perforation. The occurrence of this happened at a mean of 27 months (range 3-54, median 23.5) after surgery. Eleven cases had a surgical resolution, with a laparoscopic approach in 9 of them and laparotomy on 2. In all cases, a perforated ulcer in the jejunal side of the gastrojejunal anastomosis was found. There was no mortality or morbidity associated with surgery. Conclusions: In our experience the occurrence of perforated marginal ulcer after a LGBP develops in a small percentage of patients. The laparoscopic approach is of choice, presenting a low morbidity and mortality. Smoking was present in most patients.
\end{abstract}

Key words: Perforated ulcer, marginal ulcer, gastric bypass.

\section{Resumen}

Introducción: La perforación de una úlcera marginal es un evento grave que suele requerir una reoperación y se asocia a morbimortalidad. El manejo de estos pacientes es debatido y complejo. Objetivo: Describir una serie de pacientes operados de bypass gástrico laparoscópico (BPGLP) que evolucionaron con una úlcera

* Recibido el 18 de marzo de 2014 y aceptado para publicación el 5 de mayo de 2014.

Los autores no refieren conflictos de interés.

Correspondencia: Dr. Mauricio Gabrielli N.

megabrielli@uc.cl 
marginal perforada. Material y Método: Se revisaron retrospectivamente las fichas de pacientes operados de BPGL los últimos 10 años y que evolucionaron con una úlcera marginal perforada. Se analizaron las características clínicas, de tratamiento y morbimortalidad perioperatoria. Resultados: Durante este período se operaron 2.095 pacientes de BPGLP, 12 de los cuales presentaron una úlcera marginal perforada, correspondientes a 10 mujeres y 2 hombres. El promedio de edad fue 39 años (21-60) y el índice de masa corporal (IMC) promedio al momento de la primera cirugía fue 34 (29,3-38,6). Diez pacientes tenían hábito tabáquico activo al momento de la perforación. La ocurrencia de esta sucedió en promedio a los 27 meses (rango 3-54, mediana 23,5) de la cirugía. En 11 casos la resolución fue quirúrgica, mediante abordaje laparoscópico en 9 y laparotomía en 2. En todos los casos se encontró una úlcera perforada en la vertiente yeyunal de la gastroyeyuno anastomosis. No hubo mortalidad ni morbilidad asociada a la cirugía. Conclusiones: En nuestra experiencia la ocurrencia de úlcera marginal perforada post BPGLP se desarrolla en un bajo porcentaje de pacientes. El abordaje laparoscópico es de elección, presentando una baja morbimortalidad. El hábito tabáquico estuvo presente en la mayoría de los pacientes.

Palabras clave: Úlcera perforada, úlcera marginal, bypass gástrico.

\section{Introducción}

El BPGLP constituye una de las cirugías bariátricas de elección, siendo la más frecuente realizada en el mundo ${ }^{1}$, esto dado a sus buenos resultados y baja tasa de complicaciones ${ }^{2,3}$. La tasa de éxito a largo plazo en la mantención de la baja de peso y resolución de comorbilidades iguala o supera a otros procedimientos comúnmente realizados como la manga o banda gástrica ${ }^{2,4}$. Como todo procedimiento quirúrgico presenta complicaciones generales y específicas a la cirugía. Dentro de estas últimas destaca la aparición de úlceras marginales, las cuales corresponden a úlceras que aparecen en la mucosa yeyunal cerca de una anastomosis gastro-yeyunal y que puede extenderse a esta, entidad descrita por Berg hace más de un siglo ${ }^{5}$.

Las úlceras marginales post BPGLP tienen una incidencia aún desconocida considerando que en muchas ocasiones son asintomáticas y la realización de endoscopias de rutina no es la práctica habitual. Una revisión reciente informa cifras entre un 0,6 a $25 \%$, situándose la mayoría de los estudios analizados en esa revisión en cifras intermedias ${ }^{6}$. Se han descrito muchos factores relacionados al desarrollo de éstas, tales como el tipo de material de sutura, el tamaño y posición de la bolsa gástrica, la presencia de Helicobacter pylori, diabetes mellitus, enfermedad cardiovascular o el uso de anti inflamatorios no esteroidales (AINES) o tabaco, presentando distintos grados de asociación y resultados contradictorios en las distintas series ${ }^{6}$.

Cuando presentan sintomatología lo más frecuente es el dolor abdominal, náuseas y vómitos, describiéndose también sangrado y disfagia ${ }^{7}$. Una complicación poco frecuente es la perforación, cuadro grave que suele presentarse como dolor abdominal súbito asociado a compromiso del paciente y clínica de abdomen agudo. El análisis de este pequeño grupo de pacientes ha sugerido que el tabaco sería uno de los principales factores asociados, no estando bien esclarecido aún el perfil que presentan estos pacientes ni el tratamiento más adecuado.

El objetivo del trabajo es describir los pacientes operados de BPGLP que evolucionaron con una úlcera marginal perforada, sus características clínicas, de tratamiento y morbimortalidad perioperatoria.

\section{Material y Método}

Estudio retrospectivo y descriptivo de todos los pacientes operados de BPGLP que evolucionaron con una úlcera marginal perforada en los últimos 10 años en nuestra institución (2003-2013). La técnica quirúrgica empleada fue similar en todos los casos, siendo descrita previamente ${ }^{2}$. A todos los pacientes se les realizó test de ureasa en la endoscopia preoperatoria de rutina, realizándose tratamiento de erradicación si el resultado era positivo. Se recopilaron los datos concernientes al sexo, edad, comorbilidades, tipo y fecha BPGLP, evolución ponderal, presencia de factores de riesgo para úlcera marginal o la presencia de estas en endoscopias previas. En cuanto a la patología de urgencia se consideró la forma de presentación, exámenes preoperatorios, tipo de cirugía, hallazgos quirúrgicos, manejo y presencia de complicaciones postoperatorias y estadía hospitalaria. Se definió como complicación postoperatoria a las ocurridas hasta los 30 días del postoperatorio.

\section{Resultados}

Durante el período de estudio se operaron 2.095 pacientes de BPGLP, de los cuales 12 consultaron al servicio de urgencia por una úlcera marginal perforada. El grupo estaba compuesto por 2 hombres y 10 mujeres y el promedio de edad fue de 39 años (21-60). El peso promedio previo al BPGLP fue de 
$95 \mathrm{~kg}(70-122)$ y el IMC promedio $34,4(30,1-38,6)$ (Tabla 1). Dos de estos pacientes tenían diabetes mellitus tipo 2, tres hipertensión, 9 dislipidemia y 2 reflujo gastroesofágico (Tabla 2).

El tiempo transcurrido entre el BPGLP y la ocurrencia de la perforación fue en promedio de 27 meses (rango 3-54, mediana 23,5). El peso promedio de los pacientes al momento de la perforación fue de $68,7 \mathrm{~kg}(50-88)$ y el IMC promedio $25,7(21,4-30,1)$, presentando un porcentaje de pérdida de exceso de peso promedio de $68,4 \%$ (Tabla 3 ). Once pacientes presentaban hábito tabáquico activo, 2 pacientes se encontraban consumiendo AINES, uno de ellos asociado a corticoides (prednisona) y antibiótico (amoxicilina) en el contexto de una afección dental. Sólo 2 pacientes se encontraban tomando omeprazol y dos pacientes tenían el antecedente de una cirugía reciente (Tabla 4). La primera paciente se realizó una hernioplastía incisional laparoscópica (hernia laparotomía media infraumbilical por cesáreas) 1 mes antes, recibiendo AINES endovenosos durante su hospitalización y siendo dada de alta al segundo día postoperatorio. El segundo paciente tenía el antecedente de insuficiencia renal crónica sin necesidad de terapia dialítica, enfermedad vascular periférica (portador de prótesis aorto bi-ilíaca desde el 2008) y poseer un aneurisma tóraco-abdominal, motivo por el cual fue sometido 5 semanas antes a una reparación de aorta torácica por vía endovascular, con buena evolución postoperatoria y sin recibir AINES durante la hospitalización. Sólo una paciente (la misma sometida a la hernioplastía incisional) tenía el diagnóstico de úlcera marginal realizado 10 meses antes, momento en el cual presentó un cuadro de epigastralgia y posteriormente vómitos y melena. La endoscopia realizada durante esa hospitalización reveló la presencia de dos úlceras marginales y una esofagitis grado B de Los Ángeles. El test de ureasa fue negativo. Desde ese momento se mantuvo tomando omeprazol hasta el momento de la perforación.

En todos los pacientes el cuadro se presentó como abdomen agudo y si bien el cuadro clínico era sugerente, el diagnóstico se confirmó con la realización de una tomografía computada de abdomen y pelvis con contraste endovenoso. En 11 pacientes se realizó una cirugía de urgencia, con abordaje laparoscópico en 9 de ellos y por laparotomía en 2. La elección del abordaje fue a elección del cirujano que se encontraba de turno, dependiendo de su experiencia en cirugía laparoscópica avanzada. En los 11 pacientes se encontró una úlcera perforada en la vertiente yeyunal de la gastro-yeyuno anastomosis realizándose rafia primaria con puntos separados de seda en 10 pacientes (Figura 1). En uno de los pacientes operado por vía abierta, se instaló una sonda $\mathrm{T}$ a través de
Tabla 1. Características principales de los pacientes

\begin{tabular}{|lc|}
\hline Variable & $\mathbf{n}$ \\
Pacientes & 2.095 \\
Casos & 12 \\
Hombres & 2 \\
Mujeres & 10 \\
Edad promedio & $39(21-60)$ \\
Peso promedio pre BPGLP $(\mathrm{kg})$ & $95(70-122)$ \\
IMC promedio pre BPGLP & $34,4(30,1-38,6)$ \\
\hline
\end{tabular}

BPGLP: bypass gástrico laparoscópico, IMC: índice de masa corporal, kg: kilógramos.

Tabla 2. Comorbilidades de los pacientes

\begin{tabular}{|ll|}
\hline Variable & n \\
Diabetes mellitus 2 & 2 \\
Hipertensión & 3 \\
Dislipidemia & 9 \\
RGE & 2 \\
\hline
\end{tabular}

RGE: reflujo gastro-esofágico.

Tabla 3. Características de los pacientes al momento de la perforación

\begin{tabular}{|lc|}
\hline Variable & n \\
Tiempo transcurrido (meses) & $27(3-54)$ \\
Peso promedio 2 ${ }^{\text {a }}$ cirugía $(\mathrm{kg})$ & $68,7(50-88)$ \\
IMC promedio $2^{\text {a }}$ cirugía & $25,7(21,4-30,1)$ \\
$\%$ PEP & $68,4 \%$ \\
\hline
\end{tabular}

$\%$ PEP: porcentaje de pérdida de exceso de peso, $2^{\text {a }}$ : segunda.

Tabla 4. Presencia de factores de riesgo para úlcera marginal al momento de la perforación

\begin{tabular}{|lc|}
\hline Variable & n \\
Tabaquismo activo & 11 \\
Uso de AINES & 2 \\
Uso de corticoides & 1 \\
Cirugía previa & 2 \\
No uso de omeprazol & 10 \\
\hline
\end{tabular}

AINES: anti inflamatorios no esteroidales. 


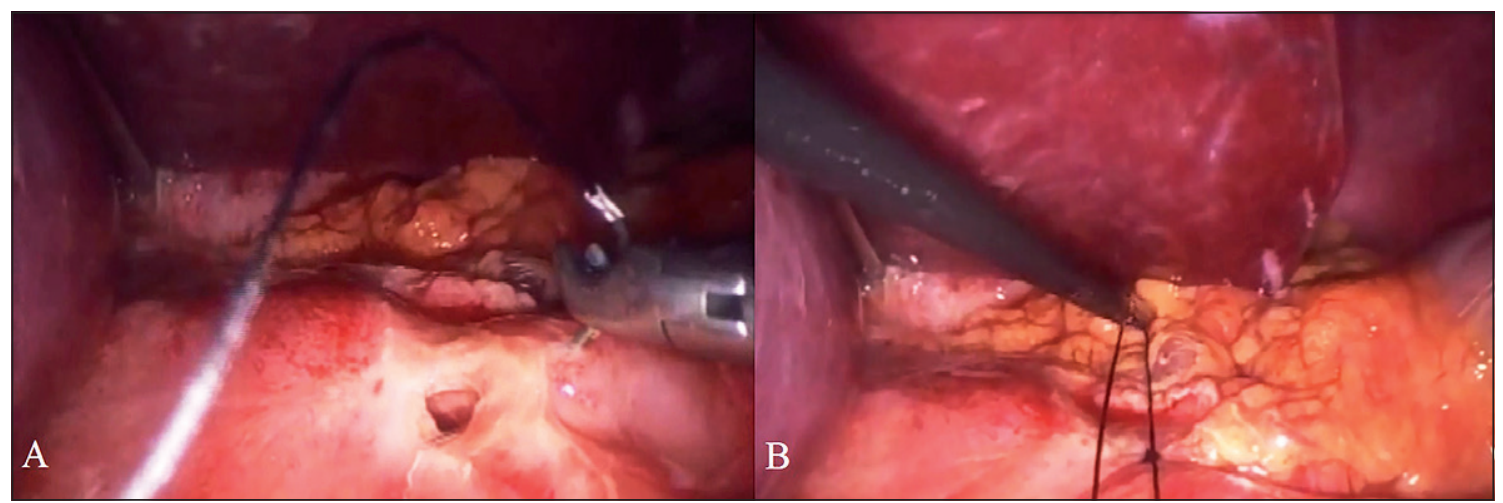

Figura 1. Reparación laparoscópica de una úlcera marginal perforada. Sutura laparoscópica de úlcera marginal post bypass gástrico laparoscópico perforada. En A se observa la úlcera rodeada de fibrina, adyacente a la gastro-yeyuno anastomosis, sobre el asa de yeyuno. En $\mathbf{B}$ el resultado final luego de anudado el primer punto de seda.

la perforación, teniendo una buena evolución posterior. Cabe mencionar que este caso fue el primer paciente de la serie.

La paciente en que se realizó manejo conservador presentaba un cuadro de menor magnitud, su tomografía computada sugería una perforación contenida a nivel de la gastro-yeyuno anastomosis. Se decidió en conjunto tratamiento médico, presentando una buena evolución posterior y siendo dada de alta al octavo día de hospitalización. En 10 pacientes se utilizó drenaje en el postoperatorio y en 3 se realizó una $\mathrm{Rx}$ con medio de contraste hidrosoluble previo a la realimentación. No hubo morbilidad ni mortalidad en esta serie, presentando una estadía hospitalaria de 6,4 días (3-11) (Tabla 5).

\section{Discusión}

Hoy es reconocido que las úlceras marginales post BPGLP han dejado de ser un problema insignificante. Si bien la mayoría de los estudios muestran cifras de prevalencia menores al $10 \%^{8-14}$, existen informes que hablan de hasta un $34 \%$ en pacientes sintomáticos ${ }^{15}$, cifra que también variaría dependiendo del momento en que se realice la endoscopia, ya que el riesgo sería mayor en el período postoperatorio temprano que en el seguimiento a largo plazo ${ }^{11,16}$. Debido a esto, se han estudiado diferentes factores que inciden en el desarrollo de esta patología, nombrándose múltiples condiciones que se asocian a esta complicación.

El tabaquismo y el uso de AINES han sido descritos como factores de riesgo en varias series ${ }^{9,15,17}$, así como también la existencia de Helicobacter pylori, y la presencia de un mayor número de células parietales; ya sea por una bolsa gástrica de mayor
Tabla 5. Manejo quirúrgico de los pacientes y evolución postoperatoria

\begin{tabular}{|lc|}
\hline Variable & n \\
\hline Resolución quirúrgica & 11 \\
Laparoscopia & 9 \\
Laparotomía & 2 \\
\hline Uso de drenaje postop. & 10 \\
Control radiológico postop. & 3 \\
Morbilidad & 0 \\
\hline Mortalidad & 0 \\
Estadía hosp. promedio (días) & $6,4(3-11)$ \\
\hline
\end{tabular}

Postop: postoperatorio, hosp: hospitalaria.

tamaño o por la existencia de una fístula gastrogástrica ${ }^{9,13,15}$. La realización de la gastro-yeyuno anastomosis con material de sutura no reabsorbible; manteniendo cierto gado de inflamación local persistente $^{9}$, la isquemia de la anastomosis, secundaria a una mayor tensión al realizar una anastomosis ante-cólica o secundario al uso excesivo de suturas mecánicas al confeccionar la bolsa ${ }^{18}$ o por ser el paciente en sí portador de factores de riesgo para insuficiencia vascular (diabetes, enfermedad arterial, etc.), son factores descritos que también pueden incidir en la aparición y perforación de una úlcera marginal post $\mathrm{BPGLP}^{6}$.

Como en muchas patologías, probablemente la etiología es multifactorial, presentando los pacientes varios de los factores de riesgo más habituales, aunque se ha descrito que hasta un $20 \%$ no tendría ningún factor de riesgo identificable ${ }^{19}$.

El tratamiento de la úlcera marginal no perforada consiste en el control de los factores de riesgo, 
la erradicación de Helicobacter pylori y el uso de inhibidores de la bomba de protones, con lo cual la gran mayoría presenta regresión completa de las lesiones ${ }^{11,13}$ y sólo en casos muy específicos se plantea la cirugía, como cuando se pesquisa algún factor de riesgo corregible por esta vía (por ejemplo una fístula gastro-gástrica) o en el caso de úlceras marginales refractarias, en las cuales se pueden plantear otras opciones como la resección y re-confección de la anastomosis ${ }^{7,15,20}$. Hay que tener en cuenta que un pequeño porcentaje puede presentar recurrencia de la úlcera marginal una vez tratada, presentando una mayor probabilidad de sangrado o perforación y, por lo tanto, la necesidad de tratamiento quirúrgico"

La perforación es un evento grave que suele requerir una reoperación, por lo que se deben hacer todos los esfuerzos para evitar esta complicación. La probabilidad de perforación de una úlcera marginal es baja, describiéndose una prevalencia entre un 0,4 a $0,8 \% \%^{7,15,17,19-23}$, concordando lo informado en este trabajo con la literatura. Al ser un grupo pequeño de pacientes, los factores asociados al desarrollo de esta complicación se encuentran menos estudiados, sin embargo, algunos tienden a repetirse en las distintas series. Por ejemplo, el hábito tabáquico ha sido una constante, presentándose en 18 de 35 pacientes con úlcera marginal perforada post bypass gástrico en el trabajo publicado por Félix y cols. ${ }^{19}$, siendo esta la serie más grande publicada hasta el momento. En nuestra serie, 11 pacientes presentaban hábito tabáquico activo al momento de la perforación, posicionándose éste como uno de los principales posibles factores de riesgo asociados al desarrollo de esta complicación. Se han descritos varios efectos agresores de la nicotina sobre el tubo digestivo tales como promover un aumento en la secreción de ácido, facilitar la infección de Helicobacter pylori y aumento de la liberación tanto de pepsinógeno como de vasopresina, favoreciendo de esta forma el desarrollo de úlcera péptica ${ }^{24}$. Otro factor asociado a perforación sería el uso de AINES, presentándose en nuestra serie en sólo dos pacientes (10 pacientes en el estudio de Félix et al. $)^{19}$. Cabe mencionar que dos pacientes habían tenido una cirugía reciente previo a la ocurrencia de la perforación, uno de los cuales recibió AINES durante esa hospitalización y probablemente haya consumido los primeros días post alta, sumándose de esta forma al estrés quirúrgico el efecto de estos medicamentos.

Diez de nuestros pacientes no se encontraban usando omeprazol al momento de la perforación. En nuestra institución a todos los pacientes luego de operados se les indica omeprazol por unos 2-3 meses, manteniéndolo posteriormente según análisis de cada caso en particular y preferencia del tratante. En general, no está normado la dosis ni el tiempo que estos pacientes deben usar los inhibidores de la bomba de protones, planteándose que el uso a permanencia para prevenir este tipo de complicaciones no sería una buena opción, dado la baja prevalencia, costo y efectos adversos del uso crónico. Por lo tanto, lo recomendado es el uso selectivo, considerar a los pacientes operados de BPGLP como una población especial que requiere evitar permanentemente estos factores de riesgo como el tabaco y uso de AINES, recurriendo a los inhibidores de la bomba de protones cada vez que se presente la necesidad, ya sea frente a una afección banal como una infección del tracto respiratorio superior o un estrés quirúrgico.

Nuestra experiencia ha demostrado que el tratamiento quirúrgico por vía laparoscópica de estos pacientes es una alternativa muy útil y segura, sin morbi-mortalidad en nuestra serie, sin embargo, experiencias similares han informado morbilidad y mortalidad del $30 \%$ y $10 \%$, respectivamente ${ }^{23}$. La técnica empleada, consistente en aseo peritoneal y posterior rafia de la lesión en un solo plano con material de sutura no reabsorbible. Esto nos parece adecuado y suficiente, sin tener que requerir parches de epiplón ${ }^{7,17,21,22,25-28}$ o ligamento ${ }^{23}$ o tener que incluir el uso de sellantes de fibrina sobre la sutura ${ }^{23,26}$.

Finalmente, la perforación de una úlcera marginal post BPGLP es un hecho poco frecuente, sin embargo, debemos estar atentos a su ocurrencia ya que la mayoría de los pacientes presentan factores de riesgo fácilmente identificables y modificables, lo que permitiría prevenir esta grave complicación que implica una cirugía y eventualmente morbi-mortalidad.

\section{Referencias}

1. Buchwald H, Oien DM. Metabolic/bariatric surgery worldwide 2011. Obes Surg. 2013;23:427-36.

2. Guzmán S, Manríquez M, Raddatz A, Norero E, Salinas J, Achurra P, y cols. Experiencia de 18 años de cirugía de obesidad en la Pontificia Universidad Católica de Chile. Rev Med Chile 2013;141:553-61.

3. Escalona A. Complicaciones quirúrgicas en bypass gástrico laparoscópico. Rev Chil Cir. 2006;58:97-105.

4. Franco JVA, Ruiz PA, Palermo M, Gagner M. A review of studies comparing three laparoscopic procedures in bariatric surgery: sleeve gastrectomy, Roux-en-Y gastric bypass and adjustable gastric banding. Obes Surg. 2011;21:1458-68.

5. Berg AA. The etiology of gastric ulcer and an outline of its therapeutics. Med Record 1898;54:150-4.

6. Coblijn UK, Goucham AB, Lagarde SM, Kuiken SD, van Wagensveld BA. Development of ulcer disease after Roux-en-Y gastric bypass, incidence, risk factors, and patient presentation: a systematic review. Obes Surg. 2014;24: 299-309. 
7. Moon RC, Teixeira AF, Goldbach M, Jawad MA. Management and treatment outcomes of marginal ulcers after Roux-en-Y gastric bypass at a single high volume bariatric center. Surg Obes Relat Dis. (en prensa).

8. Higa KD, Boone KB, Ho T. Complications of the laparoscopic Roux-en-Y gastric bypass: 1,040 patients-what have we learned? Obes Surg. 2000;10:509-13.

9. Azagury DE, Abu Dayyeh BK, Greenwalt IT, Thompson CC. Marginal ulceration after Roux-en-Y gastric bypass surgery: characteristics, risk factors, treatment, and outcomes. Endoscopy 2011;43:950-4.

10. Bhayani NH, Oyetunji TA, Chang DC, Cornwell EE 3erd, Ortega G, Fullum TM. Predictors of marginal ulcers after laparoscopic Roux-en-Y gastric bypass. J Surg Res. 2012;177:224-7.

11. Csendes A, Burgos AM, Altuve J, Bonacic S. Incidence of marginal ulcer 1 month and 1 to 2 years after gastric bypass: a prospective consecutive endoscopic evaluation of 442 patients with morbid obesity. Obes Surg. 2009;19:135-8.

12. Garrido Jr. AB, Rossi M, Lima Jr. SE, Brenner AS, Gomes Jr. CAR. Early marginal ulcer following Roux-en-Y gastric bypass under proton pump inhibitor treatmentprospective multicentric study. Arq Gastroenterol. 2010;47:130-4.

13. Rasmussen JJ, Fuller W, Ali MR. Marginal ulceration after laparoscopic gastric bypass: an analysis of predisposing factors in 260 patients. Surg Endosc. 2007;21:1090-4

14. Sapala JA, Wood MH, Sapala MA, Flake Jr. TM. Marginal ulcer after gastric bypass: a prospective 3-year study of 173 patients. Obes Surg. 1998;8:505-16.

15. El-Hayek K, Timratana P, Shimizu H, Chand B. Marginal ulcer after Roux-en-Y gastric bypass: what have we really learned? Surg Endosc. 2012;26:2789-96.

16. Csendes A, Torres J, Burgos AM. Late marginal ulcers after gastric bypass for morbid obesity. Clinical and endoscopic findings and response to treatment. Obes Surg. 2011;21:1319-22.

17. Kalaiselvan R, Exarchos G, Hamza N Ammori BJ. Incidence of perforated gastrojejunal anastomotic ulcers after laparoscopic gastric bypass for morbid obesity and role of laparoscopy in their management. Surg Obes Relat Dis. 2012;8:423-8.

18. Capella JF, Capella RF. Staple disruption and marginal ulceration in gastric bypass procedures for weight reduction. Obes Surg. 1999;6:44-9.

19. Felix EL, Kettelle J, Mobley E, Swartz D. Perforated marginal ulcers after laparoscopic gastric bypass. Surg Endosc. 2008;22:2128-32.

20. Patel RA, Brolin RE, Gandhi A. Revisional operations for marginal ulcer after Roux-en-Y gastric bypass. Surg Obes Relat Dis. 2009;5:317-22.

21. Sasse KC, Ganser J, Kozar M, Watson RW, McGinley $\mathrm{L}$, Lim D, et al. Seven cases of gastric perforation in Roux-en-Y gastric bypass patients: what lessons can we learn? Obes Surg. 2008;18:530-4.

22. Wendling MR, Linn JG, Keplinger KM, Mikami DJ, Perry KA, Melvin WS, et al. Omental patch repair effectively treats perforated marginal ulcer following Roux-en-Y gastric bypass. Surg Endosc. 2013;27:3849.

23. Lublin M, McCoy M, Waldrep DJ. Perforating marginal ulcers after laparoscopic gastric bypass. Surg Endosc. 2006;20:51-4.

24. Thomas GA, Rhodes J, Ingram JR. Mechanism of disease: nicotine-a review of its actions in the context of gastrointestinal disease. Nat Clin Pract Gastroenterol Hepatol. 2005;2:536-44.

25. Wheeler AA, de la Torre RA, Fearing NM. Laparoscopic repair of perforated marginal ulcer following Rouxen-Y gastric bypass: a case series. J Laparoendosc Adv Surg Tech. 2011;21:57-60.

26. Binenbaum SJ, Dressner RM, Borao FJ. Laparoscopic repair of a free perforation of a marginal ulcer after Roux-en-Y gastric bypass: a safe alternative to open exploration. JSLS 2007;11:383-8.

27. Bramkamp M, Muller MK, Wildi S, Clavien PA, Weber M. Perforated ulcer at the gastrojejunostomy: laparoscopic repair after Roux-en-Y gastric bypass. Obes Surg. 2006;16:1545-7.

28. Chin EH, Hazzan D, Sarpel U, Herron DM. Laparoscopic repair of a perforated marginal ulcer 2 years after gastric bypass. Surg Endosc. 2007;21:2110. 